\title{
The Risk of SARS-CoV-2 Infection Among Healthcare Workers
}

\author{
Bahar Madran¹ (D), Şiran Keske1 (D), Yeşim Beşli 2 (D), Issmail Bozkurt³ (D), Önder Ergönül4 (D), \\ 1 Infection Control, American Hospital, Istanbul, Turkey \\ 2 Department of Clinical Microbiology, American Hospital, Istanbul, Turkey \\ 3 Quality Control Unit, American Hospital, Istanbul, Turkey \\ 4 Department of Infectious Diseases and Clinical Microbiology, Koç University School of Medicine, İstanbul, Turkey
}

\begin{abstract}
Objective: We aimed to describe the risks of health care workers (HCWs) getting COVID-19, and analyze the transmission routes and dynamics of the infection.

Methods: This is a prospective observational study. We screened $624 \mathrm{HCWs}$ from April 1 to May 15, 2020, in a hospital with 300 hospital beds, in Istanbul, Turkey. All the HCWs working at high-risk areas (COVID wards, emergency departments, and intensive care units) were routinely screened every four weeks. The HCWs were grouped as high, moderate, low and none according to their risk of infection.

Results: Out of 1300 total HCWs, 42 (3.2\%) were diagnosed as COVID-19, 39 (3\%) were confirmed by polymerase chain reaction (PCR) test. Among 42 symptomatic cases, $26(62 \%)$ HCWs were hospitalized, mainly because of isolation needs, $62 \%$ received hydroxychloroquine (HCQ) alone, 11.5\% HCQ + azithromycin, and 11.5\% favipiravir only. All survived. We detected nine asymptomatic cases out of 550 HCWs (1.6\%) in our screening for antibody levels. In none of the nine asymptomatic HCWs, SARS-CoV-2 RNA was not detected by PCR. In multivariate analysis for detecting the risk factors of the SARS-CoV-2 infection, working in high-risk areas (OR:5.2, CI:1.99-13.6, p=0.001), and not to use proper personal protective equipment (PPE) (OR:5.9, CI:1.66-21.2, $\mathrm{p}=0.006)$ increased the risk of infection.

Conclusion: Routine screening of asymptomatic HCWs with antibody tests might be useful, but its effectiveness was limited. The HCWs working in high-risk areas had significantly higher risk. The strict use of appropriate PPE was effective in prevention.
\end{abstract}

Keywords: SARS-CoV-2, healthcare workers, SARS-CoV-2 infection screening

\section{INTRODUCTION}

A new coronavirus (coronavirus (SARS-CoV-2) appeared at the end of 2019 in China and spread widely throughout the world (1). The coronavirus disease 2019 (COVID-19) is a new respiratory disease, and its pandemic has affected more than seven million people all around the world (2). The first COVID-19 case in Turkey was detected on March 11, 2020. Ministry of Health announced the first death because of COVID-19 on
Corresponding Author: Bahar Madran

E-mail:

bmadran19@ku.edu.tr

Received: August 8, 2020 Accepted: August 30, 2020 Published: August 31, 2020

\section{Suggested citation:}

Madran B, Keske Ş, Beşli Y, Bozkurt I, Ergönül Ö. The Risk of SARS-CoV-2 Infection Among Healthcare Workers. Infect Dis Clin Microbiol 2020; 2: 54-60.

DOI: 10.36519/idcm.2020.0019 
March 17, 2020. Since then, until June, 13, 2020, the number of Turkey cases increased to 175.000 (3).

The main route of SARS-CoV-2 transmission is human to human (4); therefore, one of the most vulnerable population groups for infection are healthcare workers (HCWs) $(5,6)$. To prevent patient-to-HCW transmission, adherence to isolation rules, and improvement in the awareness of HCWs is important (7). However, asymptomatic cases can lead to a spread of infection among HCWs in hospitals; therefore, follow up of the high-risk contacts and screening studies are necessary to detect the asymptomatic HCWs cluster in healthcare settings (8). We aimed to describe the risks of COVID-19 infection among HCWs. Our study will shed light on the transmission routes and dynamics of the infection.

\section{METHODS}

\section{Study Design and Participants}

This is a prospective observational study. We screened 624 HCWs from April 1 to May 15, 2020, in a hospital with 300 hospital beds, in Istanbul, Turkey. We included HCWs ( $\mathrm{n}=74$ ) who had COVID-19 symptoms and suspicious exposure to the COVID-19 cases. The symptomatic HCWs were followed by daily phone calls and checked for the requirement of hospitalization. The case definition of the World Health Organization (WHO) was used for the diagnosis of COVID-19, including probable and confirmed cases (9). The confirmation was defined as the positive polymerase chain reaction (PCR) test result of COVID-19 infection, while the probable

\section{HIGHLIGHTS}

- Describing the risks of COVID-19 infection among health care workers (HCWs) is critical.

- The effectiveness of routine screening of asymptomatic HCWs is limited.

- Strict adherence to basic principles of infection control is critical for the prevention of COVID-19 infection among HCWs.

- Transmission of infection out of the hospital should be considered. case was defined as a suspect case whose SARSCoV-2 PCR test result was negative.

A questionnaire form was completed for epidemiologic, demographic information, and unintentional exposures to pathogens transmitted by droplets or aerosols according to standard precautions. The clinical, laboratory and radiologic information were collected from medical records.

\section{Definition of Risk Category}

The HCWs were grouped as high, moderate, low and none according to their risk of infection (10). The high-risk group included the HCWs, who had contact with a COVID-19 patient without any personal protective equipment (PPE) or a standard mask; the moderate-risk group included the HCWs, who had contact with a COVID-19 patient without protective goggles or with standard mask instead of N95 mask in aerosol-generating procedures. The low-risk group included the ones, who had contact with a COVID-19 patient without gown and glove. The HCWs with full PPE while caring for a COVID-19 patient were not defined in the risk group.

\section{Laboratory studies}

Nasopharyngeal and oropharyngeal swabs were obtained when HCWs reported respiratory symptoms or fever. If there was a suspected exposure, samples were obtained seven days after their contact. Samples were tested for SARSCoV-2 by real-time polymerase chain reaction (PCR) in the laboratory of Koç University Hospital in Istanbul. For PCR, mediated amplification and detection of $\mathrm{B}-\beta \mathrm{COV}$ (target E gene) specific RNA and SARS CoV-2 (target S gene), QIAamp® Viral RNA Mini Kit (Qiagen, Hilden, Germany) for nucleic acid extraction and then the RealStar@ SARS-CoV-2 RT-PCR Kit 1.0 (Altona Diagnostics, Hamburg, Germany) on Rotor-Gene® Q5/6 plex Platform (Qiagen, Hilden, Germany) were used.

The screening was performed using antibody tests. For qualitative detection of COVID-19 IgM/ IgG antibodies, Ovios ${ }^{\circledR}$ COVID-19 IgM/IgG Rapid Cassette test (Citus Diagnostic, Canada), a membrane-based immune assay having a \%73.3-96.4 agreement with PCR was used. For the symptomatic cases, all the tests were repeated using Elecsys $₫$ 


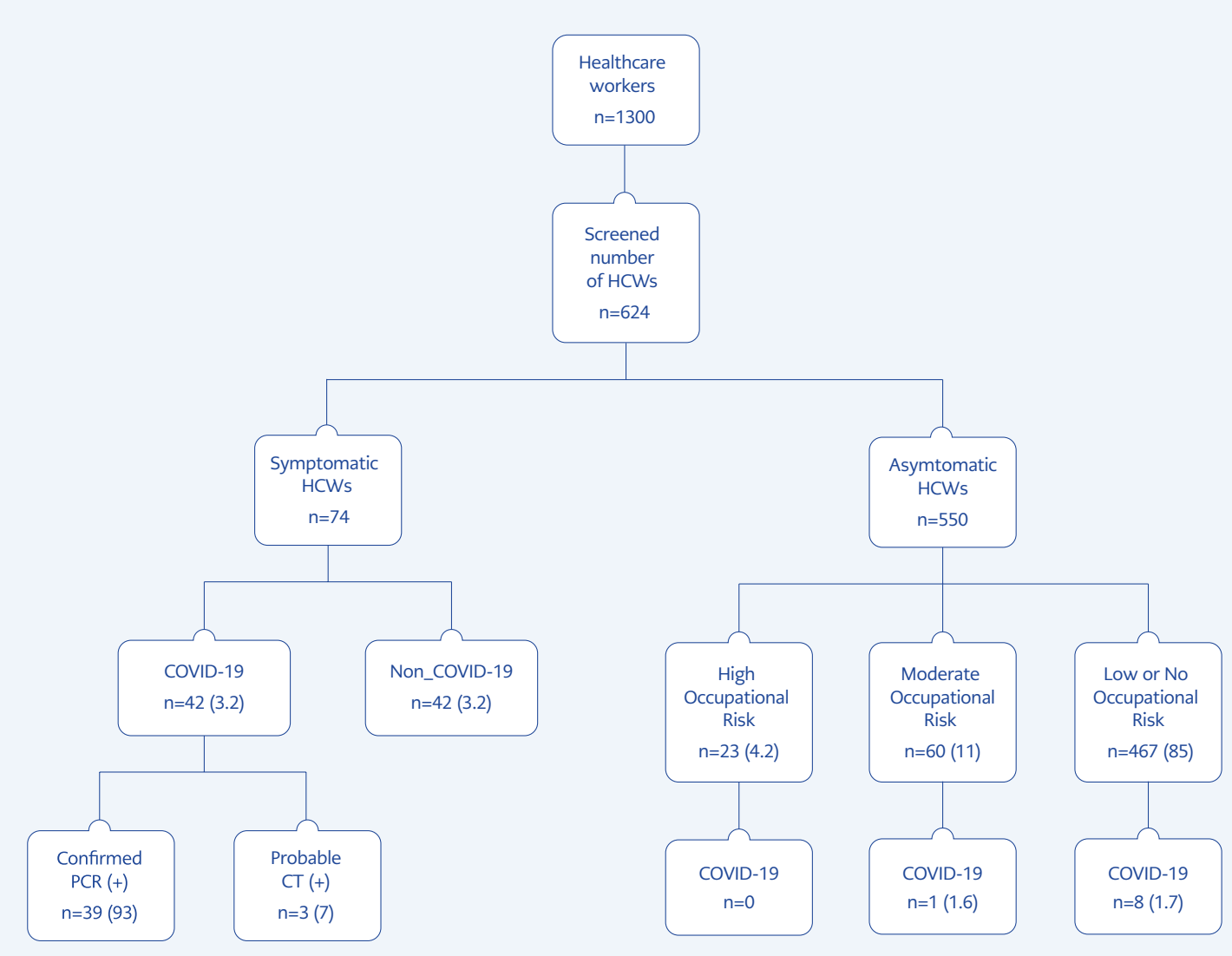

Figure 1. Symptomatic and asymptomatic HCWs.

(Roche, $\mathrm{CH}$ ). The HCWs with positive antibody tests were tested by PCR and confirmed.

\section{Statistical Analysis}

In univariate analysis, chi-square was used for categorical variables and a t-test was used for continuous variables. Multivariate analysis was performed for the risk of infection. Logistic regression was applied by using backward selection. Independent variables were duration of working, working in a high risk area, gender, being a nurse, no effective PPE, and using public transport. Statistical significance was set as $\mathrm{p}<0.05$ and Stata 16v was used. All collected data was securely stored in an electronic database.

\section{RESULTS}

We present our results in two major groups: first, features of the symptomatic cases, and second, the findings of screening studies. Out of 1300 total HCWs, 42 (3.2\%) were diagnosed as COVID-19, 39 (3\%) were confirmed by PCR test, remaining three HCWs were diagnosed based on CT results and clinical findings (Figure 1 and Figure 2). Among 42 symptomatic cases, 26 (62\%) HCWs were hospitalized, mainly because of isolation needs. Among 26 hospitalized HCWs, 85\% received treatment for COVID-19, 62\% hydroxychloroquine (HCQ) alone, $11.5 \%$ HCQ + azithromycin, and 11.5\% favipiravir only. All the symptomatic HCWs had mild or moderate disease; none had severe or critical disease.

We detected nine asymptomatic cases out of 550 HCWs (1.6\%) in our screening for antibody levels. In none of the nine asymptomatic HCWs, SARS-CoV-2 RNA was not detected by PCR. The rate of asymptomatic HCWs among the infected ones was 18\% (9 out of 51). There was no antibody positivity in the 


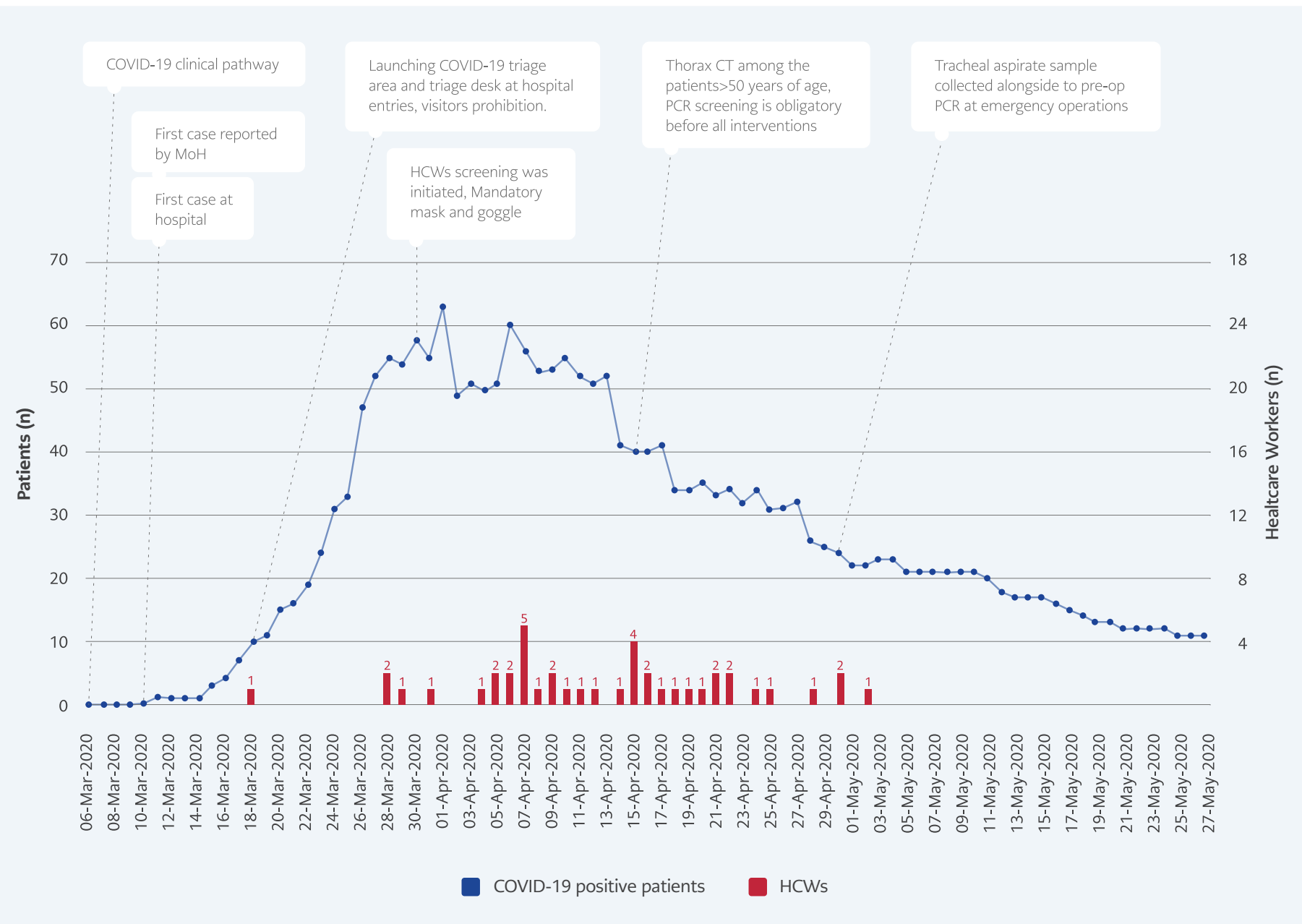

Figure 2. The relationship between the number of COVID-19 positive patients and HCWs

high risk group, one (1.6\%) in moderate, and eight $(1.7 \%)$ in low or no risk group (Figure 1).

The mean duration of work was shorter among the infected HCWs than the non-infected ones (6.2 vs. 9.1 years, $p=0.019$, Table 1). Among the infected HCWs, the proportion of physicians was lower ( $4 \%, p=0.02) .43 \%$ of the infected HCWs were not caregivers, such as physicians, nurses, and medical asisistants ( $p=0.01$, Table 1 ). The infection was significantly more common among the high-risk group ( $16 \%$ vs. $3.5 \%, p<0.001$, Table 1). Among the infected group, 33\% acquired the infection from the com munity (Table 1). Among the HCWs, $67 \%$ acquired the infection within the hospital, and $18 \%$ of them acquired the infections with no healthcare-associated activities. In multivariate analysis, working in a high risk area (OR:5.2, CI:1.99-13.6, $\mathrm{p}=0.001$ ), and not using proper PPE (OR:5.9, CI:1.66-21.2, p=0.006) increased the risk of infection.

\section{DISCUSSION}

The HCWs worked at the frontline during the pandemic, and therefore they had high risk of infection. We detected 3.2\% of SARS-CoV-2 infection among 1300 HCWs working in our hospital. In our study, the last screening was performed ten weeks after detecting the first case in the hospital, which could be compared with the studies from Germany and UK. We found only nine HCWs out of 550 were (1.6\%) asymptomatic, a study from Germany reported a similar result (11). The rate of asymptomatic infections among HCWs was reported as 3\% in the United 
Table 1. Analysis of the risk factors for SARS-CoV-2 infection ( $n=624)(\%)$

\begin{tabular}{|c|c|c|c|}
\hline & Infected $n=51(8 \%)$ & No infected n=573 (92\%) & $\mathrm{p}$ \\
\hline Mean age (min-max) & $32.2(\mathrm{sd}=7.8,23-60)$ & $34.2(\mathrm{sd}=9.9,20-71)$ & 0.08 \\
\hline Mean duration of work & $6.2(s d=6.0)$ & $9.1(s d=8.7)$ & 0.019 \\
\hline Gender & & & 0.535 \\
\hline Male & $21(41)$ & $209(37)$ & \\
\hline Female & $30(59)$ & $599(63)$ & \\
\hline \multicolumn{4}{|l|}{ Occupation } \\
\hline Physician & $2(4)$ & $89(15)$ & 0.02 \\
\hline Nurse & $18(35)$ & $246(43)$ & 0.29 \\
\hline Caregiver & $9(18)$ & $70(12)$ & 0.26 \\
\hline $\begin{array}{l}\text { Others (radiology technicians, } \\
\text { administrative staff, security) }\end{array}$ & $22(43)$ & $151(26)$ & 0.01 \\
\hline \multicolumn{4}{|c|}{ Occupational risk within the hospital } \\
\hline High & $8(16)$ & $20(3.5)$ & $<0.001$ \\
\hline Moderate & $5(10)$ & $63(11)$ & 0.79 \\
\hline Low or None & $38(74)$ & $490(85)$ & 0.037 \\
\hline \multicolumn{4}{|l|}{ Covid-19 } \\
\hline Confirmed & $48(94)$ & NA & \\
\hline Probable & 3 & NA & \\
\hline \multicolumn{4}{|l|}{ Source } \\
\hline Community & $17(33)$ & NA & \\
\hline Hospital environment & $34(67)$ & NA & \\
\hline Healthcare associated & $28(82)$ & NA & \\
\hline Social & $6(18)$ & NA & \\
\hline
\end{tabular}

Table 2. Univariate and multivariate analyses for SARS CoV-2 infection ( $n=51)$

\begin{tabular}{|l|c|c|c|c|c|c|}
\hline \multirow{2}{*}{ SARS CoV-2 infection ( $n=51)$} & \multicolumn{3}{|c|}{ Univariate analysis } & \multicolumn{3}{c|}{ Multivariate analysis } \\
\cline { 2 - 7 } & Odds ratio & $\begin{array}{c}\text { Confidence } \\
\text { interval (95\%) }\end{array}$ & $p$ & Odds ratio & $\begin{array}{c}\text { Confidence } \\
\text { interval (95\%) }\end{array}$ & $p$ \\
\hline Duration of work & 0.95 & $0.91-0.99$ & 0.043 & - & - & - \\
\hline Working in COVID wards & 2.74 & $1.46-5.15$ & 0.002 & 5.2 & $-1.99-13.6$ & - \\
\hline Female gender & 0.83 & $0.46-1.49$ & 0.536 & - & - & - \\
\hline Nurse & 0.73 & $0.40-1.32$ & 0.29 & - & - & - \\
\hline No PPE & 3.7 & $1.36-10.1$ & 0.01 & 5.9 & $1.66-21.2$ & 0.006 \\
\hline
\end{tabular}


Kingdom (UK) (8). In a study from the Netherlands, after screening HCWs with PCR, $1 \%$ of HCWs were infected with SARS-CoV-2 (12). This rate was lower than other studies, but the study was performed within two weeks of the first case of the pandemic. In studies from Wuhan, at the beginning of the outbreak, the infection rate among HCWs was 1.1\% (13), and in a recent study, the infection rate among HCWs was $1.8 \%$ (14). In a study from New Jersey, the rate of antibody positivity among 121 intensive care units was reported as $0.83 \%(15)$. Considering such a low rate of antibody level among the HCWs indicates that infection control practices were successful in preventing the infection among HCWs.

In our study, among the infected HCWs, the mean duration of work was shorter (6.2 vs. 9.1 days, $\mathrm{p}=0.019$ ). The infection rate was higher among the HCWs working at the high-risk areas: COVID-19 wards, the intensive care unit, and the emergency department. ( $16 \%$ vs $3.5 \%, p<0.001)$. In multivariate analysis, working in high-risk areas (OR:5.2, CI:1.99-13.6, $\mathrm{p}=0.001)$, and not using proper PPE (OR:5.9, CI:1.66-21.2, $\mathrm{p}=0.006$ ) were found to be associated with infection.

However, in screening, there was no antibody positivity among the HCWs who had a high risk of exposure, who had contact with a COVID-19 patient without any personal protective equipment (PPE) or standard mask, one (1.6) among the moderate group and eight (1.7\%) among low or no risk group. Similar findings were reported from Wuhan (13).

The low infection rate among HCWs could be explained by the strict adherence to infection control measures. The PPE advice was changed according to the department and daily new cases in the hospital (Figure 2). Using surgical masks (equivalent to ASTM level 2), protective goggles, latex gloves (equivalent to AAMI level 2), waterproof/longsleeved cloth gowns, and disposable round caps was mandatory. The high-contagion areas additionally included particulate respirators (N95 mask), waterproof/long-sleeved cloth gowns, leakproof goggles, disposable/spaceman type round caps, and latex gloves. When needed to use extra PPEs in low-contagion areas, especially during some aerosol proceeding procedures, for instance, nasopharyngeal sampling HCWs obtained all type extra equipment (respirators, leakproof goggles, etc.). Shoe cover has never used in our hospital that is assessed risky practice for contamination of hands by infection control committee members. Bodysuits were kept as an alternative to waterproof/long-sleeved cloth gown but were not preferred by HCWs.

\section{CONCLUSION}

In our institutions, both symptomatic and asymptomatic infection among HCWs was lower than expected. The rapid diagnosis and isolation of infected HCWs could help to protect HCWs. Routine screening of asymptomatic HCWs with antibody tests might be useful, but its effectiveness was limited. The antibody screening test should be performed according to the epidemic curve of the COVID-19 in the community. The HCWs working in high-risk areas had significantly higher risk. The strict use of appropriate PPE was effective in prevention.

Conflict of Interest: The authors have no conflict of interest to declare.

Financial Disclosure: The authors declared that this study has received no financial support.

Acknowledgement: We thank to Ayşegül Ergönül for her support in language
Author Contributions: Concept - Ö.E.; Design - B.M., Ö.E.; Supervision - Ö.E., B.M.; Data Collection and/or Processing - B.M., Ş.K., Y.B., I.B.; Analysis and/or Interpretation - Ö.E.; Literature Review - B.M., Ş.K., Y.B., İ.B.; Writer - B.M., Ö.E., Ş.K.; Critical Reviews - Ö.E..
Ethical Approval: Koç University Ethical Committee for Research IRB1.066.

Peer-review: Externally peer-reviewed

Bouopda Tamo et al. 


\section{REFERENCES}

1 Zhu N, Zhang D, Wang W, Li X, Yang B, Song J, et al. A Novel Coronavirus from Patients with Pneumonia in China, 2019. N Engl J Med. 2020;382:727-33.

2 Worldometer. COVID-19 Coronavirus Pandemic. Coronovirus cases 2020 (cited 2020 June 6). Available from: https://www. worldometers.info/coronavirus/

3 Republic of Turkey Ministry of Health. Current situation in Turkey (cited 2020 June 6). Available from: https://covid19bilgi.saglik.gov.tr

4 Hoehl S, Rabenau H, Berger A, Kortenbusch M, Cinatl J, Bojkova $\mathrm{D}$, et al. Evidence of SARS-CoV-2 infection in returning travelers from Wuhan, China. N Engl J Med 2020;382: 1278-80.

5 Luo Y, Trevathan E, Qian Z, Li Y, Li J, Xiao W, et al. Asymptomatic SARS-CoV-2 infection in household contacts of a healthcare provider, Wuhan, China. Emerg Infect Dis 2020; 26.

6 Weissman DN, de Perio MA, Radonovich LJ, Jr. COVID-19 and risks posed to personnel during endotracheal intubation. JAMA 2020; 10.1001/jama.2020.6627.

7 Wong SC, Kwong RT, Wu TC, Chan JWM, Chu MY, Lee SY, et al. Risk of nosocomial transmission of coronavirus disease 2019: an experience in a general ward setting in Hong Kong. J Hosp Infect 2020; 105: 119-27.

8 Rivett L, Sridhar S, Sparkes D, Routledge M, Jones NK, Forrest S, et al. Screening of healthcare workers for SARS-CoV-2 highlights the role of asymptomatic carriage in COVID-19 transmission. Elife 2020; 9: e58728.

9 The World Health Organization. Global surveillance for COVID-19 caused by human infection with COVID-19 virus 2020 (cited 2020 April 20). Available from: https://www.who. int/publications-detail/global-surveillance-for-human-infection-with-novel-coronavirus-(2019-ncov)
10 National Health Commission \& National Administration of Traditional Chinese Medicine. Diagnosis and Treatment Protocol for Novel Coronavirus Pneumonia. 2020 (cited 2020 June 10). Available from: https://www.chinadaily.com.cn/ pdf/2020/1. Clinical. Protocols. for.the.Diagnosis.and. Treatment.of.COVID-19.V7.pdf.

11 Korth J, Wilde B, Dolff S, Anastasiou OE, Krawczyk A, Jahn M, et al. SARS-CoV-2-specific antibody detection in healthcare workers in Germany with direct contact to COVID-19 patients. J Clin Virol.2020; 128: 104437.

12 Kluytmans-van den Bergh MFQ, Buiting AGM, Pas SD, Bentvelsen RG, van den Bijllaardt W, van Oudheusden AJG, et al. Prevalence and clinical presentation of health care workers with symptoms of coronavirus disease 2019 in 2 Dutch hospitals during an early phase of the pandemic. JAMA Netw Open 2020; 3: e209673.

13 Lai X, Wang M, Qin C, Tan L, Ran L, Chen D, et al. Coronavirus Disease 2019 (COVID-2019) infection among health care workers and implications for prevention measures in a tertiary hospital in Wuhan, China. JAMA Netw Open. 2020; 3: e209666.

14 Xu X, Sun J, Nie S, Li H, Kong Y, Liang M, et al. Seroprevalence of immunoglobulin $\mathrm{M}$ and $\mathrm{G}$ antibodies against SARS-CoV-2 in China. Nat Med 2020; 26: 1193-95.

15 Mughal MS, Kaur IP, Patton CD, Mikhail NH, Vareechon C, Granet KM. The Prevalence of SARS-CoV-2 IgG Antibodies in Intensive Care Unit (ICU) Healthcare Personnel (HCP) and its Implications - A Single-Center, Prospective, Pilot Study. Infect Control Hosp Epidemiol 2020: 1-6. 